\title{
Apoptotic Effects of the B Subunit of Bacterial Cytolethal Distending Toxin on the A549 Lung Cancer Cell Line
}

\author{
Hajar Yaghoobi ${ }^{1}$, Mojgan Bandehpour ${ }^{1,2,3 *}$, Bahram Kazemi ${ }^{1,2,3}$
}

\begin{abstract}
Cytolethal distending toxin (CDT) is a secreted tripartite genotoxin produced by many pathogenic gram-negative bacteria. It is composed of three subunits, $\mathrm{CdtA}, \mathrm{CdtB}$ and $\mathrm{CdtC}$, and $\mathrm{CdtB}$-associated deoxyribonuclease (DNase) activity is essential for the CDT toxicity. In the present study, to design a novel potentially antitumor drug against lung cancer, the possible mechanisms of cdtB anticancer properties were explored in the A549 human lung adenocarcinoma cell line. A recombinant plasmid pcDNA3.1/cdtB was constructed expressing $\mathrm{CdtB}$ of human periodontal bacterium Aggregatibacter actinomycetemcomitans and investigated for toxic properties in A549 cells and possible mechanisms. It was observed that plasmid pcDNA3.1/cdtB caused loss of cell viability, morphologic changes and induction of apoptosis. Furthermore, measurement of caspase activity indicated involvement of an intrinsic pathway of cell apoptosis. Consequently, the recombinant plasmid pcDNA3.1/cdtB may have potential as a new class of therapeutic agent for gene therapy of lung cancer.
\end{abstract}

Keywords: Cytolethal distending toxin - genotoxin - recombinant plasmid - Aggregatibacter actinomycetemcomitans

Asian Pac J Cancer Prev, 17, Cancer Control in Western Asia Special Issue, 299-304

\section{Introduction}

Cancer is one of the serious causes of death. according to WHO statistics in 2012, an estimated 8.2 million people died from cancer worldwide. Lung cancer is the most common leading cause of cancer with 1.6 million deaths (Bermudes et al., 2002 ,Yildirim et al., 2015), which can be due to occurrence of metastasis in the early stages, delayed diagnosis and rapid progression of the cancer (Qiu et al., 2012). Lung cancer divides in two subgroups including small cell lung cancer (SCLC) and non-small cell lung cancer (NSCLC) by which the latter is more prevalent (Govindan et al., 2006). Conventional anticancer therapies such as surgery, chemotherapy and radiation therapy for people with advanced lung cancers is much less effective and therefore the development of more efficient strategies is appear to be necessary (Zhou et al., 2015). Gene therapy is a good choice for the handling of several diseases including cancer (Sun et al., 2007). Bacterial toxins can interfere in proliferation control, apoptosis and differentiation or even can cause cell death. In the latter strategy, cells are directly killed via the protein expressed by the toxin genes (Patyar et al., 2010).Cytolethal distending toxin (CDT) is a bacterial genotoxin produced by variety of gram-negative bacteria, like Actinobacillus actinomycetemcomitans that can damage tissue at distinct anatomical sites. Secreted tripartite protein is encoded by an operon containing three genes namely $\operatorname{cdt} \mathrm{A}, \mathrm{cdtB}$ and cdtC. A and C subunits has binding to cell surface and $B$ subunit possesses a catalytic role. In contrast to the A and $\mathrm{C}$ subunits that have a high sequence diversity probably due to lifestyle and host niche of producing bacteria, subunit B has high sequence and structure homology to DNase I that bring about DNA damage after its delivery into cells. CDT degrades nuclear DNA in cells and also inhibits the proliferation of cells by induction of $\mathrm{G} 2$ cell cycle arrest and thereby apoptosis inevitably is raised. However, some believe that B subunit would be enough for ultimate performance (Bezine et al., 2014). Herein, we have investigated anticancer property of the catalytic subunit B of Aggregatibacter actinomycetemcomitans through transfection of A549 human lung adenocarcinoma cells using recombinant plasmid pcDNA3.1/CdtB.

\section{Materials and Methods}

\section{Cell Cultures and Transfection}

A549, human lung adenocarcinoma cells were maintained in DMEM/F12 media (Gibco) supplemented with antibiotics $(50 \mathrm{IU} / \mathrm{ml}$ penicillin, $50 \mathrm{microgram} \mathrm{g} / \mathrm{ml}$ streptomycin) and $5 \%$ fetal bovine serum (FBS). The cells were incubated at $37^{\circ} \mathrm{C}$ in $5 \% \mathrm{CO} 2$ humidified incubator for growth. Cell were transfected with lipofectamin 2,000 (Invitrogen) according to manufacture order.

${ }^{1}$ Department of Biotechnology, School of Medicine, ${ }^{2}$ Cellular and Molecular Biology Research Center, ${ }^{3}$ Department of Biotechnology, School of Advanced Technologies in Medicine, Shahid Beheshti University of Medical Sciences, Tehran, Iran. *For Correspondence: Bandehpour@gmail.com 


\section{Plasmid construction}

Aggregatibacter actinomycetemcomitans $852 \mathrm{bp}$ fragment encoding catalytic subunit $\mathrm{CdtB}$ was synthesized according to the nucleotide sequences available from the National Center for Biotechnology Information (NCBI) database and subcloned into pcDNA3. 1+ vector using XhoI and XbaI restriction enzymes and made pcDNA3.1/ cdtB. Construct was selected in E. coli Top10 and Plasmid DNA was isolated using an Accuprep ${ }^{\circledR N a n o-P l u s ~ P l a s m i d ~}$ Mini Extraction Kit (BIONEER, Korea). Construct was verified by PCR using universal and specific primers and DNA sequencing. Primers used in this study are listed in Table 1.

Cytolethal distending toxin (CDT) transcription assay by $R T-P C R$

Twenty-four hours before transfection of cells, A549 cell line were seeded into 6 -well plates at a density of $0.6 \times 10^{6} \mathrm{cell} /$ well per well then treated with pcDNA3.1/ cdtB. After $12 \mathrm{~h}, 24 \mathrm{~h}$ and $48 \mathrm{~h}$, total RNA was extracted from similarly control and transfected cell line using Total RNA Purification Kit (Jena Bioscience, Germany), cdtB mRNA was changed to cDNA using Rocket Script RT Premix and poly-dT primer as reported by the manufacturer (BIONEER, Korea), then amplified by PCR using specific primers.

Observation of morphologic changes in intoxicated cells using invert fluorescent microscopy

A549 cells $(5 \times 105$ cells/well $)$ were seeded into 12-well plates and treated with pcDNA3.1/cdtB After 24 $\mathrm{h}, 48 \mathrm{~h}$ and $72 \mathrm{~h}$ the cells were analyzed and photographed by JULTM Smart fluorescent cell analyzer .

\section{In vitro Cytotoxicity assay}

\section{Evaluation of cell proliferation by MTT Assay}

The number of viable A549 cell line after transfection was evaluated by the MTT (3-[4, 5-methylthiazol-2-yl] -2, 5-diphenyl-tetrazolium bromide) assay in the manner of Sargent and Taylor(Sargent and Taylor, 1989).Briefly, cells were seeded into 96 -well plate $(2 \times 104$ cells/well $)$ and twenty-four hours kept for attachment. Set of plated cells was transfected in triplicate and this treatment was carried out on cells with minimum of $75 \%$ Confluence, after 24,48 , and 72 hours. $10 \mu \mathrm{l}$ of MTT $(5 \mathrm{mg} / \mathrm{ml})$ was added on to each well and incubated for $3 \mathrm{~h}$ at $37^{\circ} \mathrm{C}$ in a $5 \% \mathrm{CO} 2$ incubator. After completing the incubation the MTT solution was removed and $100 \mu$ of dimethyl sulfoxide (DMSO) was added to each well to solubilize the formazan. The absorbance at $570 \mathrm{~nm}$ was determined by ELISA reader. The reduction of cell proliferation (\%) was calculated using the following formula: cell inhibition (\%) $=(1-\mathrm{A} 570 \mathrm{of}$ treated cells $/ \mathrm{A} 570$ of control cells $) \times 100 \%$.

Evaluation of A549 cell count and viability by trypan blue dye.

A549 cells were plated in 6 -well plates $\left(0.6 \times 10^{6} \mathrm{cell} /\right.$ well), incubated for 24 hours then were treated with pcDNA3.1/cdtB as described in the MTT assay. Following incubation, in a humidified 5\% CO2 incubator at $37^{\circ} \mathrm{C}$ for $48 \mathrm{hr}$, the supernatant pool was collected and adherent cells were trypsinized and collected. Cell viability was performed by the dye exclusion test with $0.5 \%$ trypan blue using a hemocytometer. Both viable and nonviable cells were counted. A minimum of 200 cells was counted for each data point in a total of five microscopic fields.

\section{Necrosis and apoptosis assay}

\section{DNA Fragmentation analysis}

Briefly, the cells were plated in 6-well plates $\left(0.6 \times 10^{6} \mathrm{cell} / \mathrm{well}\right)$, incubated for 24 hours, and then transfected with pcDNA3.1/cdtB or pcDNA3.1 for 48 hours in the absence of serum. After washing the cells twice with PBS, adherent cells were trypsinized and were harvested then lysed in $200 \mu \mathrm{l}$ cell lysis buffer by stirring with a wide-bore pipette tip. $10 \mu \mathrm{R}$ RAase A solution was added, mixed well and incubated at $37^{\circ} \mathrm{C}$ for 120 minutes. After adding phenol / chloroform and centrifugation, the supernatant was collected and treated with $1000 \mu \mathrm{l}$ of $100 \%$ ethanol for overnight. Centrifuge was performed at $-4{ }^{\circ} \mathrm{C}$ for 10 minutes at $13,000 \mathrm{rpm}$. The supernatant was discarded and treated with $100 \mu \mathrm{l} 75 \% \mathrm{EtOH}$ by gently mixing. After centrifugation (2 minutes at 13000rpm), the supernatant was discarded and the pellet was air dried. The DNA pellet was dissolved in $30 \mu \mathrm{L}$ TE buffer (100 mM Tris-Cl pH7.4 and 10 mM EDTA pH8.0) and incubated for 1 hour at $37^{\circ} \mathrm{C}$. The fragmented DNA was resolved on 2\% agarose gels and electrophoresed for 180 minutes at $30 \mathrm{~V}$ and the bands were detected by UV light and documented by photography.

Terminal deoxyribonucleotidyl transferase-mediated dUTP nick end labeling (TUNEL) assay.

Table 1. List of Primers Used to Confirm Cloning and Expiration cdt B

\begin{tabular}{ll}
\hline Primer & Sequence \\
\hline Specific (CdtB) & F: 5'-ACCATGCAATGGGTAAAGCAATTA- 3' \\
Specific (CdtB) & R: 5'-CACGAACAAAACTAACAGGAAAAT- 3' \\
Universal pcDNA3.1 & F: 5'-TAATACGACTCACTATAGG- 3' \\
Universal pcDNA3.1 & R: 5'-TAGAGGCACAGTCGG- 3' \\
RT-PCR(Cdt B) & F: 5'-CAACAACACAATTCCAACCC-3' \\
RT-PCR(Cdt B) & R: 5'-GGCGATACCTGTCCATTCTT-3' \\
RT-PCR(B-actin) & F: 5'-GCATGGGTCAGAAGGATTC-3' \\
RT-PCR(B-actin) & R: 5'-GGCTACTTAGGGTGAGGATG-3' \\
\hline
\end{tabular}




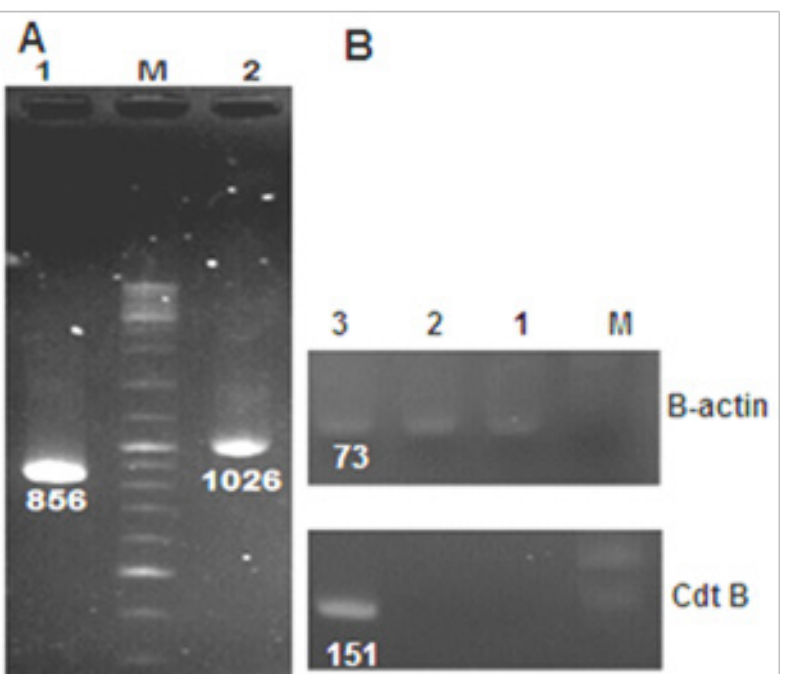

Figure 1. Verify Cloning cdtB into pcDNA3.1. and Expression of CdtB in Transfected Cell. A: Amplification of cdtB by Specific Primer (lane1) and Universal Primer (lane2). B: Expression of cdtB in A549 Cells Identified by RT-PCR. B-actin Served as Internal Control.1: Cell, 2: Treated with pcDNA3.1, 3: Treated with pcDNA3.1/ cdtB

Tunel (terminal deoxynucleotide transferase [TdTJ-mediated digoxigenin-uridine triphosphate [dig-UTP] nick-end labeling;) was performed to measure 3'-hydroxyI ends of DNA fragments formed during programmed cell death (i.e., apoptosis). A549 cells were plated in 6-well plates and treated with pcDNA3.1/cdtB and incubated for $36 \mathrm{~h}$ at $37^{\circ} \mathrm{C}$ in a $5 \% \mathrm{CO} 2$ incubator. Control or apoptosis-induced cells were trypsinized, collected, then centrifuged (5 min, $2000 \mathrm{rpm}$ ) and resuspended in $100 \mu \mathrm{l}$ PBS (phosphate buffered saline). Cells added to the poly-L-lysine-coated slides and fixed in $4 \%$ paraformaldehyde at room temperature for $20 \mathrm{~min}$. To detect nuclei with DNA fragmentation, a TUNEL-staining method was performed using a DeadEnd ${ }^{\mathrm{TM}}$ Colorimetric TUNEL System (Promega Corporation, Madison, WI, USA) according to the manufacturer's instructions. Finally the cells on the cover slip were dehydrated, and observed under a light microscope.

\section{Cell cycle analysis}

Cells were harvested by trypsinization, suspended 2 $\times 10^{4}$ cells in $0.5 \mathrm{~mL}$ PBS and aspirated several times to obtain a mono-dispersed cell suspension with minimal cell aggregation. Then they were fixed for $20 \mathrm{~min}$ on ice with $4.5 \mathrm{~mL}$ of $70 \%$ ethanol and kept for at least $2 \mathrm{~h}$ at $4^{\circ} \mathrm{C}$. Ethanol thoroughly was decanted by centrifugation for $5 \mathrm{~min}$ at $300 \mathrm{~g}$ and subsequently resuspended in 0.5 $\mathrm{ml}$ of propidium iodide $(\mathrm{PI})$ solution $(3.8 \mathrm{mM}$ sodium citrate, $0.3 \%$ NP40, $0.05 \mathrm{mg} \mathrm{ml}-1 \mathrm{PI}, 0.02 \mathrm{mg} \mathrm{ml}-1$ RNase) in PBS for $2 \mathrm{~h}$ at $4^{\circ} \mathrm{C}$. Flow cytometric analysis was performed using a flow cytometer (Partec CyFlow Space, Germany). The distribution of cells in different cell cycle phases was analyzed using Flomax software

\section{Caspase 9 assay}

In brief, $0.6 \times 10^{6}$ cells per well of 6 -well plate was seeded and kept overnight for attachment. Next day cells were transfected with pcDNA3.1/cdtB and incubated for $36 \mathrm{~h}$. Following incubation, control and toxin treated cells were harvested resuspended in $50 \mu \mathrm{l}$ lysis buffer and
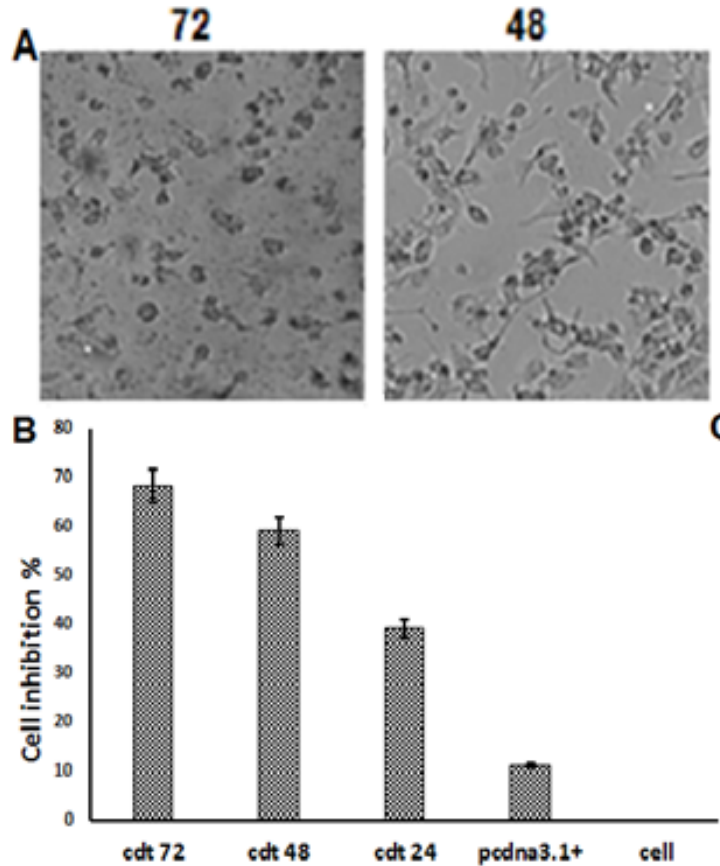

48

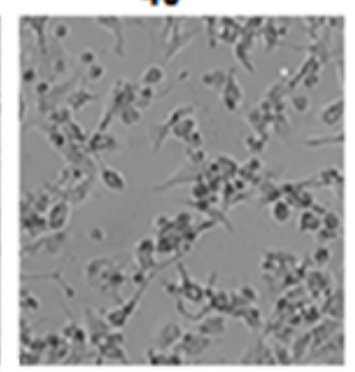

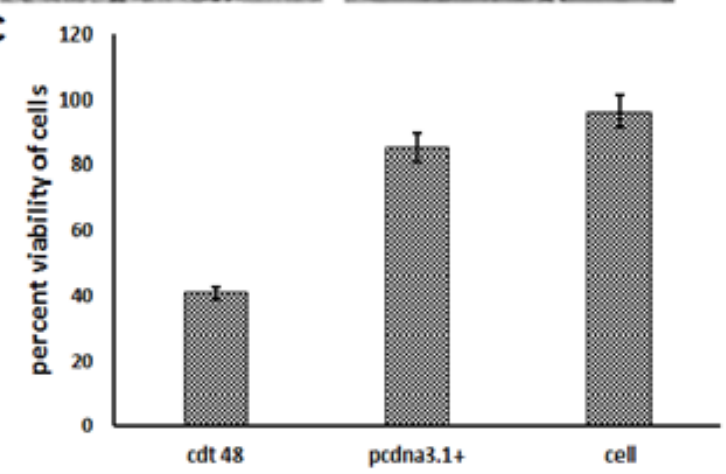

Figure 2. Inhibition of A549 Cell Proliferation by pcDNA3.1/cdtB in a Time Dependent Manner. A: Morphology of A549 Cells Transfected with pcDNA3.1/cdtB Different Time. B: Cell Viability was Determined by MTT Assay after 24 h, 48h, and 72h which Significantly Reduced in pcDNA3.1+/cdtB Group Compared to Medium Alone, pcDNA3.1+ group: $\mathrm{p}<0.01 . \mathrm{C}$ : Effect of pcDNA3.1/cdtB on A549 Cell Count. C: Graph Showing \% Cell Viability. $\mathrm{p}<0.01$. 
incubated for 10 minutes. Caspase 9 activity was measured by colorimetric assay according to the manufacturer's instructions. Abcam's Caspase 9 assay Kit (Colorimetric) prepares a simple method for assaying the activity of caspase that recognize the sequence LEHD. In this assay cleavage from labeled substrate LEHD-p-NA produces chromophore p-nitroanilide (p-NA) that the p-NA light emission can be quantified using a spectrophotometer at $405 \mathrm{~nm}$. The data were expressed as average percentages of the absorption.

\section{Statistical analysis}

Comparisons among different groups were performed with the Student t-test and one-way ANOVA using the SPSS software. $\mathrm{P}<0.01$ was defined statistically significant.

\section{Results}

Verify cloning $c d t B$ into pcDNA3.1+ and expression of CdtB in transfected cell line

The desired gene fragment has 830 nucleotides and it was successfully amplified and cloned into pcDNA 3.1(+) vector with enzymatic digestion. The construct was confirmed by colony PCR, restriction analysis and sequencing. As shown in Figure 1, successful expression of cdtB was detected in transfected A549 cell line with pcDNA3.1/cdtB by produce cDNA from cdtB mRNA using reverse transcriptase and poly-dT primers, followed by PCR using cdtB specific primers.

The effects of the Cytolethal distending toxin (CdtB) on the morphology of the cells

Upon the incubation of cells with toxin, for $24 \mathrm{~h}$, $48 \mathrm{~h}$, and $72 \mathrm{~h}$ apoptotic cells were seen. As shown in figure $2 \mathrm{~A}$ it is also clear that the most common apoptotic morphological changes observed in cells included chromatin condensation, loss of normal shape.

The effects of the Cytolethal distending toxin (c) on the A549 cell line growth and proliferation

A549, human lung adenocarcinoma cells were assayed for cell viability after treatment with the pcDNA3.1/cdtB. The results of MTT assay and trypan blue dye revealed that transfected with pcDNA3.1/cdtB significantly reduced cell viability in time-dependent manner compared to the control groups $(\mathrm{P}<0.001)$ (Fig. 2B, C)

\section{Apoptosis induction by Cytolethal distending toxin ( $C d t B)$} in human lung adenocarcinoma A549 cell line

To investigate DNA fragmentation as hallmark feature of apoptosis we studied internucleosomal DNA cleavage using DNA fragmentation analysis. In figure 3B, it appear that CdtB leads to decrease in the total genomic DNA. However, clear DNA ladder pattern was not observed in this cell line. Also, TUNEL staining revealed apoptotic cells as nuclei with considerable apoptosis was induced in transfected cells compared with (untreated cells) controls.
A

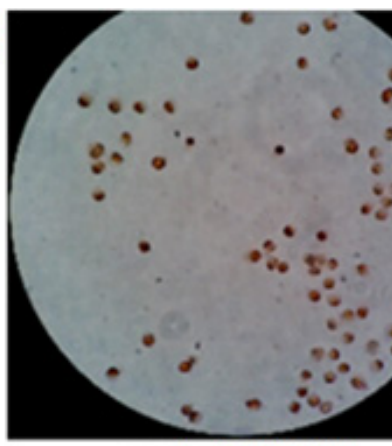

pcdna3.1+/cdt b

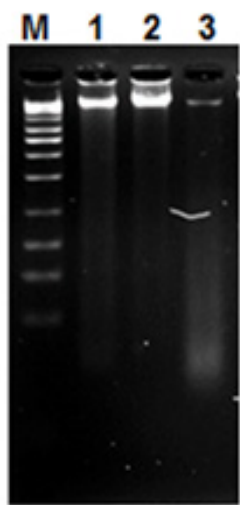

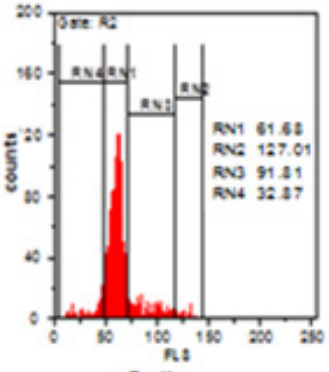

Cell

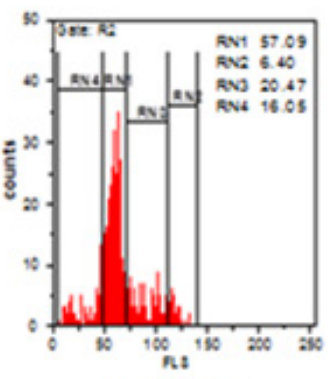

pcdna3.1+

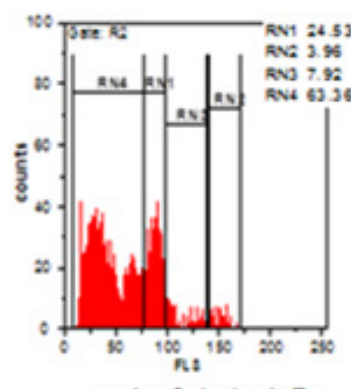

pcdna3.1+/ cdt B

Figure 3. CdtB Induces Apoptosis in A549 Cells. A: Colorimetric TUNEL Assay of A549 Cells Following 36 h Treatment. In Contrast to the Untreated Cells and pcDNA3.1+ Control, Some Apoptotic Bodies (Brown Nuclei Stained) were Seen in the Transfected Cells with pcDNA3.1+/cdtB. B: Electrophoretic Analysis of Fragmented DNA. M: Ladder, 1: Control Cells, 2: pcDNA3.1+Transfected Cell, 3: pcDNA3.1+/cdtB Transfected Cell after 72h. C: Cell Cycle Analysis of A549 Cells Transfected with pcDNA3.1+/cdtB for $48 \mathrm{~h}$. As a Control, Cells were Transfected with pcDNA3.1+ for 48h. Cells were Fixed with Ethanol, Stained by Propidium Iodide to Quantify Cellular DNA, and 20,000 Cells were Counted. 


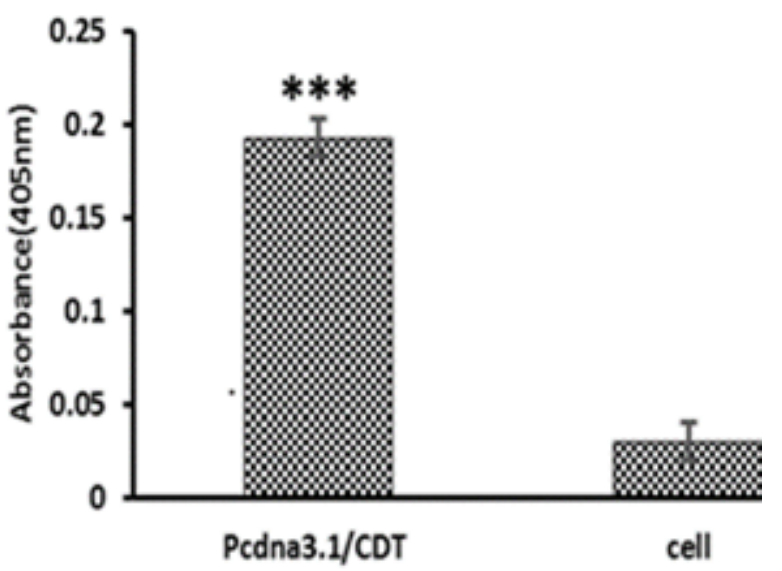

Figure 4. Changes in the Level of Caspase9 Activity at 36 h after Transfection. ***: $\mathrm{p}<0.01$

The DNA fragmentation analysis and TUNEL data were in agreement with the flow cytometry results. Cell apoptosis was evaluated using flow cytometric analysis. As shown in figure 3 the percentage of apoptotic cells significantly increased in the pcDNA3.1+/cdtB group (63.4\%), compared to the pcDNA3.1+16.5\%), or medium alone $(8.2 \%)$ groups.

\section{Caspase 9 mediated CdtB induced apoptosis}

We measured the changes in caspase 9 activity in A549 cell line after cdtB construct transfection. As shown in figure 4, a significant increase in activity of caspase- 9 was observed cdtB transfected cells after $36 \mathrm{~h}$, suggesting that subunit B is inducing apoptosis in A549 cells by intrinsic pathway via caspase-9 induction.

\section{The effect of $C d t B$ on the cycle cycle}

The effect of $\mathrm{CdtB}$ on the cell cycle progression was studied in A549 cells. Propidium iodide staining demonstrated that treatment with pcDNA3.1+/cdtB induced apoptosis however without any cell cycle arrest in the $\mathrm{G} 2 / \mathrm{M}$ phase (Figure 2). pcDNA3.1+ was used as a control showed any effect on the cell cycle and apoptosis. PI staining and flow cytometry analysis was estimated the quantification of dead cells (sub-G1) and cells in different phases of the cell cycle in the total cell population (Figure $3 \mathrm{C})$.

\section{Discussion}

During the past decade, bacteria have extensively studied for their biomedical applications in cancer therapy (Bandala et al., 2013). Live and attenuated bacteria can all be antitumor agents that have been emerged as potential strategies (Bermudes et al., 2002). Furthermore, bacterial toxins are considered as one of the promising cancer treatments (Liu et al., 2014).

For example, Shiga toxin, inhibit tumor growth in mouse xenograft models (Engedal et al., 2011). Diphtheria toxin inhibits protein synthesis, resulting in cell lysis and/or induction of apoptosis (Frankel et al., 2002). Like DT, Pseudomonas exotoxin A inhibits protein synthesis that makes it a potential candidate for targeted cancer therapy (Pastan, 1997) In addition,
Clostridium perfringens enterotoxin (CPE) displays cytotoxic activity on pancreatic cancer cells and induces tumor necrosis in vivo (Michl et al., 2001). It is being investigated for colon, breast and gastric cancers (Hough et al., 2000 , Kominsky et al., 2004).

These findings have strongly recommended the application of bacterial toxins as potential cancer therapeutic agents. Previous studies examined the antitumor potential of a recombinant plasmid DNA expressing in several cancer cell lines in which successful expression of insert and the reduction of cancer growth were observed (Mizrahi et al., 2010 ,Wang et al., 2012). Therefore, in this study, attention was focused on a plasmid DNA vector-based strategy and also evaluating the effects of cdtB derived from Aggregatibacter actinomycetemcomitans on apoptosis, cell cycle, and their underlying mechanisms in A549, a human lung adenocarcinoma cell line. Some investigators have demonstrated that CdtB-associated deoxyribonuclease (DNase) activity is responsible for Cdt-induced cell cycle arrest, suggesting that DNase activity is essential for the Cdt toxicity. For example, in several studies on Cdt from A. actinomycetemcomitans, cdtA, cdtB and cdtC genes were cloned and purified and then, function of their gene products was investigated (Saiki et al., 2001 , Nishihara and Koseki, 2004). Effect of CdtB (rcdtB) protein into gingival squamous cell carcinoma cells using a BioPORTER as an in vitro system for delivery and also effects of cdtB gene transfection with sonoporation on the viability of cells confirmed apoptosis following delivery of those molecules in cells (Nishihara and Koseki, 2004 ,Yamamoto et al., 2004).Cheng-Kuo Lai et all., have attempted to use His-tagged CdtB subunit along with chitosan/heparin nanoparticle vehicle system for delivering transfected into gastric cancer cells. The findings confirmed nanoparticle-CdtB-induced cell death of gastric cancer cells due to DSBs and G2/M cell-cycle arrest, followed by apoptosis (Lai et al., 2014). In another study, Wising C et al (2010) reported that, toxic properties of replication deficient adenovirus type 5 (Ad5) vector expressing CdtB cause loss of cell viability, morphologic changes, and cell cycle arrest on HeLa cells. In the present study cdt B was successfully amplified by PCR and cloned in pcDNA 3.1(+) vector with enzymatic digestion. The construct was confirmed by sequencing. The expression of cdtB in cells is detectable on the mRNA level. Decreased viability of A549 cells showed by our data from trypan blue and MTT test with clear difference between control cells and transfected cell with cdtB-expressing vector, and also TUNEL assay confirmed slightly increased numbers of apoptotic A549 cells after treatment. However, results of cell cycle analysis by flow cytometry did not show cdtB can induce G2 cell cycle arrest of A549 cells same previous study and But rather high levels induction of apoptosis and cell death in was observed. To gain insight into the molecular mechanism involved in cdtB induced apoptosis, the activation of caspase-9 was assessed in A549 cells. Our results confirmed activation of caspase- 9 which causes cleave inactive procaspase- 3 to active caspase-3, thereby providing a link between the mitochondria and cdt $\mathrm{B}$ induced apoptosis in A549 cells. The present findings 
provide evidence that pcDNA3.1 $(+) / \mathrm{cdtB}$ vector has a significant effect on arresting cell cycle and inducing apoptosis in A549 cells, similar to changes demonstrated in cells treated with holotoxin CDT. Therefore, cdtB may have a potentially promising application against non-small cell lung cancer. However, expression of the protein should be targeted for prevention of damage to the normal cells.

\section{Acknowledgements}

This paper was extracted from Hajar Yaghoobi PhD thesis and it was carried out in Cellular and Molecular Biology Research Center of Shahid Beheshti University of Medical Sciences. The authors declare that there is no conflict of interest.

\section{References}

Bandala C, Perez-Santos JL, Lara-Padilla E, et al (2013). Effect of botulinum toxin a on proliferation and apoptosis in the t47d breast cancer cell line. Asian Pac J Cancer Prev, 14, 891-4.

Bermudes D, Zheng LM ,King IC (2002). Live bacteria as anticancer agents and tumor-selective protein delivery vectors. Curr Opin Drug Discov Devel, 5, 194-9.

Bezine E, Vignard J ,Mirey G (2014). The cytolethal distending toxin effects on mammalian cells: A DNA damage perspective. Cells, 3, 592-615.

Engedal N, Skotland T, Torgersen ML, et al (2011). Shiga toxin and its use in targeted cancer therapy and imaging. Microb Biotechnol, 4, 32-46.

Frankel AE, Rossi P, Kuzel TM, et al (2002). Diphtheria fusion protein therapy of chemoresistant malignancies. Curr Cancer Drug Targets, 2, 19-36.

Govindan R, Page N, Morgensztern D, et al (2006). Changing epidemiology of small-cell lung cancer in the united states over the last 30 years: Analysis of the surveillance, epidemiologic, and end results database. J Clin Oncol, 24, 4539-44.

Hough CD, Sherman-Baust CA, Pizer ES, et al (2000). Large-scale serial analysis of gene expression reveals genes differentially expressed in ovarian cancer. Cancer Res, 60, 6281-7.

Kominsky SL, Vali M, Korz D, et al (2004). Clostridium perfringens enterotoxin elicits rapid and specific cytolysis of breast carcinoma cells mediated through tight junction proteins claudin 3 and 4. Am J Pathol, 164, 1627-33.

Lai CK, Lu YL, Hsieh JT, et al (2014). Development of chitosan/ heparin nanoparticle-encapsulated cytolethal distending toxin for gastric cancer therapy. Nanomedicine (Lond), $\mathbf{9}$, 803-17.

Liu CC, Yang H, Zhang LL, et al (2014). Biotoxins for cancer therapy. Asian Pac J Cancer Prev, 15, 4753-8.

Michl P, Buchholz M, Rolke M, et al (2001). Claudin-4: A new target for pancreatic cancer treatment using clostridium perfringens enterotoxin. Gastroenterology, 121, 678-84.

Mizrahi A, Hochberg A, Amiur S, et al (2010). Targeting diphtheria toxin and tnf alpha expression in ovarian tumors using the h19 regulatory sequences. Int J Clin Exp Med, $\mathbf{3}$, 270-82.

Nishihara T,Koseki T (2004). Microbial etiology of periodontitis. Periodontol 2000, 36, 14-26.

Pastan I (1997). Targeted therapy of cancer with recombinant immunotoxins. Biochim Biophys Acta, 1333, $1-6$.
Patyar S, Joshi R, Byrav DS, et al (2010). Bacteria in cancer therapy: A novel experimental strategy. J Biomed Sci, 17, 21.

Qiu Y, Peng GL, Liu QC, et al (2012). Selective killing of lung cancer cells using carcinoembryonic antigen promoter and double suicide genes, thymidine kinase and cytosine deaminase (pcea-tk/cd). Cancer Lett, 316, 31-8.

Saiki K, Konishi K, Gomi T, et al (2001). Reconstitution and purification of cytolethal distending toxin of actinobacillus actinomycetemcomitans. Microbiol Immunol, 45, 497-506.

Sargent JM ,Taylor CG (1989). Appraisal of the mtt assay as a rapid test of chemosensitivity in acute myeloid leukaemia. Br J Cancer, 60, 206-10.

Sun S, Schiller JH, Spinola M, et al (2007). New molecularly targeted therapies for lung cancer. J Clin Invest, 117, 2740-50.

Wang S, Shu JZ, Cai Y, et al (2012). Establishment and characterization of mtdh knockdown by artificial microrna interference - functions as a potential tumor suppressor in breast cancer. Asian Pac J Cancer Prev, 13, 2813-8.

Wising C, Magnusson M, Ahlman K, et al (2010). Toxic activity of the cdtb component of haemophilus ducreyi cytolethal distending toxin expressed from an adenovirus 5 vector. Apmis, 118, 143-9.

Yamamoto K, Tominaga K, Sukedai M, et al (2004). Delivery of cytolethal distending toxin $\mathrm{b}$ induces cell cycle arrest and apoptosis in gingival squamous cell carcinoma in vitro. Eur J Oral Sci, 112, 445-51.

Yildirim F, Baha A, Yurdakul AS, et al (2015). Comparison of single agent gemcitabine and docetaxel in second-line therapy for advanced stage non-small cell lung cancer in a university hospital in turkey. Asian Pac J Cancer Prev, 16, 7859-65.

Zhou W, Wang J, Man WY, et al (2015). Sirna silencing ezh2 reverses cisplatin-resistance of human non-small cell lung and gastric cancer cells. Asian Pac J Cancer Prev, 16, 2425-30. 